\title{
Platelet-Activation Factor Receptor Induces Interleukin 10 Production through STAT3 Activation in Dendritic Cells
}

\author{
Marianna M Koga ${ }^{1,2^{*}}$, Luciano R Filgueiras ${ }^{1}$, Sonia Jancar ${ }^{1}$ and Francisco J Rios ${ }^{1,3}$ \\ ${ }^{1}$ Department of Immunology, Institute of Biomedical Sciences, University of São Paulo, São Paulo, Brazil \\ ${ }^{2}$ Department of Biochemistry, Faculty of Biology and Medicine, University of Lausanne, Epalinges, Switzerland \\ ${ }^{3}$ Institute of Cardiovascular and Medical Sciences, British Heart Foundation Glasgow Cardiovascular Research Centre, University of Glasgow, Glasgow, United Kingdom
} *Corresponding author: Marianna M Koga, Department of Biochemistry, Faculty of Biology and Medicine, University of Lausanne, Switzerland, Tel: 41021692 5710/5730; Fax: 41021692 5705; E-mail: marianna.koga@unil.ch

Received date: April 21, 2017; Accepted date: May 12, 2017; Published date: May 17, 2017

Copyright: (c) 2017 Koga MM, et al. This is an open-access article distributed under the terms of the Creative Commons Attribution License, which permits unrestricted use, distribution, and reproduction in any medium, provided the original author and source are credited.

\begin{abstract}
The activation of the platelet-activating factor receptor (PAFR) is associated to a suppressor phenotype in macrophages and dendritic cells (DCs). In the present study, we investigated mechanisms underlying the production of the interleukin 10 (IL-10) through PAFR activation in murine DCs. For this purpose, BALB/c mice bone marrowderived DCs were differentiated by GM-CSF treatment and stimulated with LPS in the presence of the PAFR antagonist WEB2086. Signalling pathways downstream to TLR4 activation were investigated. We found that LPS stimulus induced PAFR ligands generation by DCs, but it did not affect the PAFR expression. The LPS-induced IL-10 production was found to be partially dependent of PAFR, since it was reduced in the presence of WEB2086. The IL-10 production through PAFR activation was independent on CREB and PPARY, as the treatment with selective inhibitors of these pathways did not affect the IL-10 production. TLR4 adaptor molecules (MyD88 and TRIF) expression, MAPK, or NF-kB (p105/50 and p65 subunits) activation pathways were also excluded, since they were not affected by the treatment with WEB2086. The blockage of PAFR by WEB2086 downregulated the STAT3 (Tyr705) phosphorylation induced by LPS. Additionally, DCs treated with STAT3 inhibitor (S3I-201) showed reduced IL-10 production to the same levels observed in DCs treated with WEB2086. The requirement of STAT3 was confirmed in PAFR-KO DCs, since the STAT3 inhibition did not affect IL-10 production by these cells. Our data show an additional molecular mechanism whereby PAFR contributes to IL-10 production in DCs and support the importance of the PAFR activation in DCs phenotype and function.
\end{abstract}

Keywords: Interleukin 10; Platelet-activating factor receptor; PAFR ligands generation; Toll-like receptor 4; Dendritic cells; Prostaglandin E2; STAT3

\section{Introduction}

The interleukin 10 (IL-10) is an anti-inflammatory cytokine secreted by different immune cells that acts as an important regulator of the inflammatory and immune responses [1]. In macrophages and myeloid dendritic cells (DCs), the IL-10 production is induced by different stimuli via pattern recognition receptors (PRRs) activation, which also triggers an inflammatory response with the production of other cytokines, such as IL-1 $\beta$, IL-12, IL-6, and so on [2]. Additionally, lipid mediators are also generated and further amplify the immune response (for either inflammatory or anti-inflammatory directions) in a fast and prompt way [3].

Platelet-activating factor (PAF) is a lipid mediator rapidly generated by the cleavage of cell membranes phospholipids as a consequence of the activation of the cytosolic phospholipase A2 ( $\left.\mathrm{CPLA}_{2}\right)$ [4]. PAF acts as an intracellular and intercellular messenger through its receptor (PAFR), which is a $\mathrm{G}$ protein-coupled receptor that according to the expression in plasma or nuclear membranes may be coupled to the $G_{i}$ or $\mathrm{G}_{\mathrm{q}}$ subunits [5]. We have recently shown that mice that were immunized in the presence of a PAFR antagonist developed an enhanced antigen-specific adaptive immune response. We also observed that vaccination of mice with dendritic cells (DCs) that had been previously treated with PAFR antagonists and stimulated with lipopolysaccharide (LPS) also induced higher antigen-specific T cell proliferation, suggesting that the activation of this receptor in the site of immunization could adjust both innate and adaptive immunity responses [6].

Mechanisms involving in the amplification of cell activation by inflammatory stimuli through PAFR are still elusive, and it may differ according to receptors assembly, activation profile and cell population. In macrophages, the PAFR activation by exogenous PAF combined with Toll-like receptor 2 or 4 (TLR2/TLR4) agonists suppressed the production of the pro-inflammatory cytokines IL-12 and IL- 6 by inhibiting NF- $\kappa B$ p65 phosphorylation. On the other hand, PAF increased NF- $\kappa$ B p105 phosphorylation, which generated p50 subunit, potentiating the LPS-induced IL-10 production [7]. In DCs, we have shown that, like in macrophages, exogenous PAF enhanced LPSinduced IL-10. Additionally, pre-treatment with PAFR antagonists reduced IL-10 production and enhanced DCs T cell priming activity in vitro. Prostaglandin E2 ( $\left.\mathrm{PGE}_{2}\right)$, a lipid mediator rapidly synthesized by the inducible cyclooxygenase (COX-2) upon LPS stimulus, was also downregulated by the blockage of PAFR. Moreover, when DCs were treated with selective COX-2 inhibitors $\mathrm{PGE}_{2}$ production was abrogated, their ability to promote $\mathrm{T}$ cell proliferation was increased to the same levels observed in the presence of PAFR antagonists, but no effects were observed in IL-10 production [8]. These data indicated that IL-10 and $\mathrm{PGE}_{2}$ induction partially occurred via PAFR activation but they also suggested the existence of another LPS-induced IL-10 
production pathway independent of the COX-2/PGE 2 axis, as $\mathrm{PGE}_{2}$ itself had been described as an inducer of the $I 110$ gene expression [9]. Accordingly, in the present work, we investigated mechanisms underlying the production of IL-10 in the context of PAFR activation. We found that TLR4 activation by LPS in DCs induces PAF synthesis that, in turn, activates its receptor and increases IL-10 production through STAT3 phosphorylation pathway.

\section{Materials and Methods}

\section{Mice}

Male BALB/c wild-type or PAFR-deficient (PAFR-KO) mice at 6 to 8 weeks old were bred and maintained at the Isogenic Breeding Unit at the Department of Immunology (Biomedical Sciences Institute University of São Paulo) and kept under specific pathogen-free conditions. All experiments performed were approved according to the Brazilian Animal Welfare Regulations (National Council for Animal Experimentation Control-CONCEA). PAFR-KO mice were originally provided by Dr. Satoshi Ishii and Dr. Takao Shimizu [10].

\section{DC generation and culture}

Mice were euthanized by $\mathrm{CO}_{2}$ exposure and bone marrow cells were collected from their femurs. Cells were seeded in $100 \mathrm{~mm}$ tissue culture dishes (BD, Franklin Lakes, NJ, USA) and cultured in RPMI 1640 medium (GIBCO, Grand Island, NY, USA), supplemented with $10 \%$ heat-inactivated fetal bovine serum (FBS - GIBCO, GO, Brazil) and Antibiotic-Antimycotic solution (1x) (GIBCO). DCs differentiation was induced by $20 \mathrm{ng} / \mathrm{mL}$ of granulocyte-macrophage colony-stimulating factor (rmGM-CSF, Peprotech, Rocky Hill, NJ, USA). Half of the medium was replaced and rmGM-CSF stimulation was renewed on the third day of culture. On the sixth day, nonadherent cells were collected, transferred to new plates, and stimulated with $1 \mu \mathrm{g} / \mathrm{mL}$ of LPS (Sigma-Aldrich, Saint-Louis, MO, USA). For some experiments, cells were pre-treated for $30 \mathrm{~min}$ with the PAFR antagonist WEB 2086 (Tocris Biosciences, Ellisville, MO, USA), COX inhibitors Indomethacin (Sigma-Aldrich) or Nimesulide (Cayman Chemical, Ann Arbor, MI, USA), CREB inhibitor KG-501, PPAR inhibitor GW9662, or STAT3 inhibitor S3I-201 (all three from SigmaAldrich). Antagonist/inhibitors concentration used in the assays were previously established as optimal by dose-response tests $[7,8]$.

\section{PAFR expression analysis}

The PAF receptor blocking peptide and a rabbit anti-PAFR monoclonal antibody (both from Cayman Chemical) were mixed in 1:1 ratio and left at room temperature for $1 \mathrm{~h}$. The mixture was $100 \mathrm{x}$ diluted in FACS buffer (PBS, 1\% Fetal Bovine Serum, 0.02\% sodium azide) and added to DCs. In parallel, DCs were incubated with only anti-PAFR antibody. After $30 \mathrm{~min}$ of incubation at $4^{\circ} \mathrm{C}$, cells were washed twice with FACS buffer and incubated with anti-rabbit IgGFITC (Poly4064) (BioLegend, San Diego, CA, USA) and anti CD11cAPC (HL3) (BD Pharmingen, San Diego, CA, USA) for $30 \mathrm{~min}$, in the dark, at $4^{\circ} \mathrm{C}$. Cells were washed twice and fixed in PBS $3.7 \%$ paraformaldehyde for $10 \mathrm{~min}$, room temperature. Cells were washed and data was acquired using a FACS Canto II flow cytometer (BD Biosciences), and analyzed by the FlowJo software (TreeStar, Ashland, OR, USA). Doublets were excluded and analyses were conducted in CD $11 \mathrm{c}^{+}$gated cells. PAFR expression was given as Median Fluorescence Intensity (MFI) and values were determined by the MFI of a-PAFR-stained cells in the presence of the blocking antibody deducted from MFI of a-PAFR-stained cells to exclude antibodyprotein non-specific complexes formation.

\section{Total lipid extraction}

After 6 days of DC differentiation, non-adherent cells were collected, washed and kept with serum free medium at the final volume of $1 \mathrm{~mL} .1 \mu \mathrm{M}$ of the serine-protease inhibitor AEBSF (A8476, Sigma-Aldrich) was added per sample and after $30 \mathrm{~min}, 1 \mu \mathrm{g} / \mathrm{mL}$ LPS was added and cells were incubated for extra $30 \mathrm{~min} .2 \mathrm{~mL}$ of ice cold methanol were added, followed by homogenization on ice. Next, $2 \mathrm{~mL}$ of dichloromethane were added per sample, tubes were sealed and vortexed vigorously. Samples were centrifuged at $2500 \mathrm{x}$ g for $10 \mathrm{~min}$. The inferior phase of samples was transferred to new tubes and aqueous phase was evaporated under nitrogen. The lipid extracts were normalized to cell number $\left(2.5 \times 10^{6}\right.$ cells $)$ and dissolved in ethanol.

\section{KB PAFR model system}

PAFR-expressing KBP and PAFR-deficient KBM cells were generated from the human epidermal cell line $\mathrm{KB}$ (which does not express functional PAFR) transduced with a retroviral vector MSCV2.1 containing the human PAFR cDNA (KBP cells) or with the empty vector (KBM cells). When PAFR of $\mathrm{KBP}$ cells is activated by its agonists such as PAF or PAF-like molecules, the intracellular $\mathrm{Ca}^{2+}$ levels are increased and IL- 8 is produced. Hence, this system allows the determination of the presence of PAFR ligands in a given lipid extract indirectly, through measurement of IL-8 production. This model was developed as previously described by Pei et al. (1998) [11]. Both KBP and KBM cells were seeded at $2 \times 10^{5}$ cells $/ \mathrm{mL}$ and kept with serumfree medium for $30 \mathrm{~min}$. Then, DC lipid extracts were added to $\mathrm{KBP} / \mathrm{KBM}$ cells for $5 \mathrm{~h}$ and supernatants were collected. As positive control for cell activation and IL-8 production, $100 \mathrm{nM}$ of CPAF (Cayman Chemical) or $10 \mathrm{nM}$ of phorbol 12-myristate 13-acetate (PMA, Sigma- Aldrich) were added to KBP and KBM cells. The KB cells were kindly provided by Prof. Jeffrey B. Travers, Wright State University, OH, USA.

\section{Cytokines and $\mathrm{PGE}_{2}$ detection}

Human IL-8 production by KBM/KBP cells or murine IL-10 production by bone-marrow derived DCs were determined using OptEIA Set ELISA kits (BD Pharmingen), and $\mathrm{PGE}_{2}$ production was measured by competitive immunoassay with the $\mathrm{PGE}_{2}$ EIA kit (Cayman Chemical) according to manufacturer's instructions.

\section{Western blot}

DCs were lysed with RIPA buffer containing sodium orthovanadate (1 $\mathrm{mM})$, sodium fluoride $(5 \mathrm{mM})$, and protease inhibitor cocktail (Roche Scientific, Indianapolis, IN, USA). Same quantities of protein were separated by $10 \%$ SDS-PAGE and transferred to polyvinylidene difluoride (PVDF) membranes (GE Healthcare, Little Chalfont, Buckinghamshire, UK). Next, non-specific binding sites were blocked with 5\% non-fat dry milk (Cell Signaling Technology, Danvers, MA, EUA) in Tris-buffered saline $0.1 \%$ Tween 20 (TBS-T) for $1 \mathrm{~h}$. After washing with TBS-T, membranes were incubated with primary antibodies against phospho-CREB (Ser133), phospho-p38 (Thr180/ Tyr204), phospho-ERK1/2 (Thr202/Tyr204), total-STAT3, phosphoSTAT3 (Tyr705), $\beta$-actin (all from Cell Signaling Technology) or phospho-NF- $\kappa$ B p105/p50 (Ser337) (SAB Signaling, College Park, MD, 
Citation: Koga MM, Filgueiras LR, Jancar S, Rios FJ (2017) Platelet-Activation Factor Receptor Induces Interleukin 10 Production through

Page 3 of 9

USA) at $4{ }^{\circ} \mathrm{C}$ overnight. Membranes were then washed and incubated with secondary antibodies goat anti -rabbit IgG or goat anti-mouse IgG conjugated to HRP (both from Cell Signaling Technology) for $2 \mathrm{~h}$. Finally, the membranes were developed using the SuperSignal West
Pico Chemiluminescent Substrate (Thermo Scientific, Rockford, IL). Densitometric analyses were performed using the ImageJ software (NIH, Bethesda, MD, USA).
A

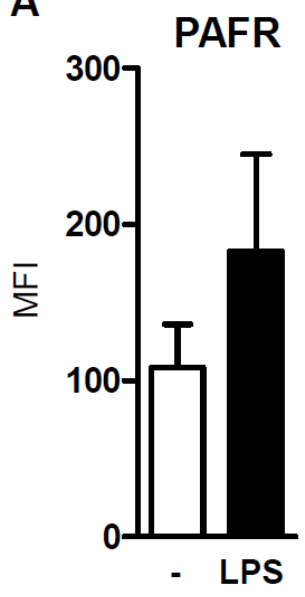

C

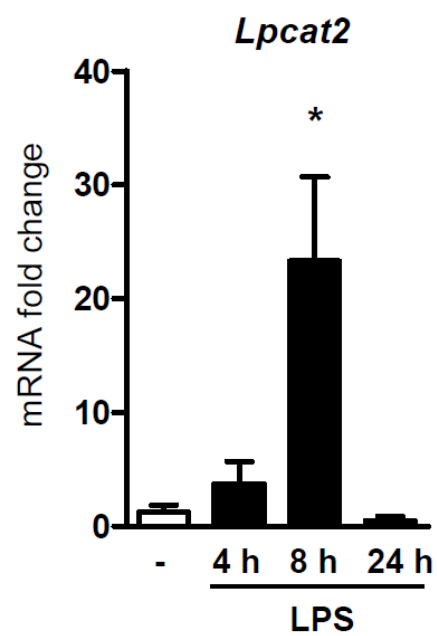

D

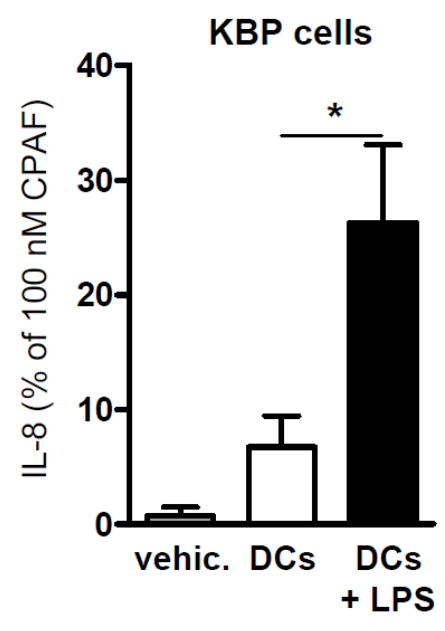

B

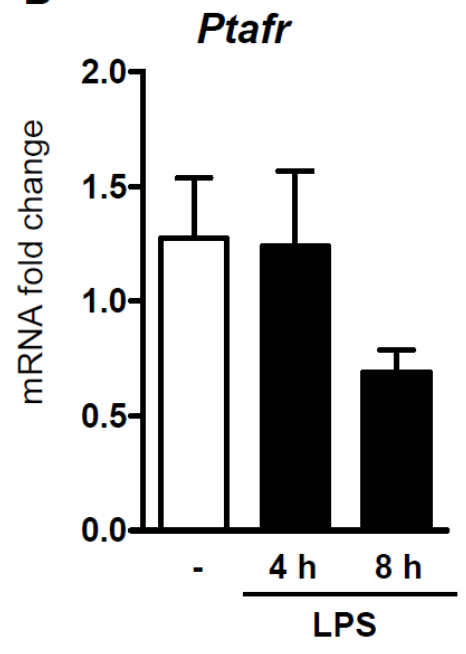

E

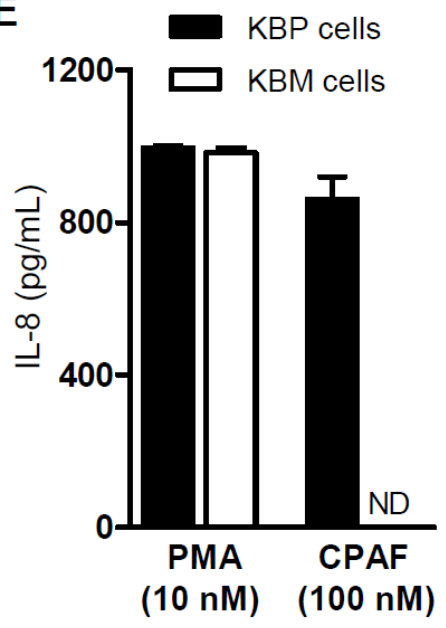

Figure 1: DCs produce PAFR ligands upon LPS stimulation. DCs were stimulated with LPS $(1 \mu \mathrm{g} / \mathrm{mL})$ for $24 \mathrm{~h}$ and stained with anti-CD11c and anti-PAFR antibodies in the presence of PAFR blocking peptide for $30 \mathrm{~min}$. PAFR expression of CD11c positive-gated cells were assessed by flow cytometry and expressed in Median Fluorescence Intensity (MFI). Results are shown mean \pm SEM of MFI values (left) and representative histograms for PAFR expression are displayed on the right. FMO (Fluorescence Minus One) control: DCs stained only with anti-CD11c (A). PAFR (Ptafr) and the inducible lyso-PAF acetyltransferase (Lpcat2) genes expression kinetic in LPS-stimulated DCs were assessed by qPCR. Data are shown as fold change of the non-stimulated control group (B-C). DCs were treated with the serine protease inhibitor AESBF $(1 \mu \mathrm{M})$ and stimulated with LPS $(1 \mu \mathrm{g} / \mathrm{mL})$ for $30 \mathrm{~min}$. Cells and supernatants were submitted to lipid extraction, resuspended in ethanol, and added to KBP cells. After $8 \mathrm{~h}$, the production of IL- 8 was assessed by ELISA (D). IL- 8 production by KBP and KBM cells stimulated with CPAF or PMA (E). Data are representative of at least three independent experiments. ${ }^{*} \mathrm{P}<0.05$ vs. non-stimulated control group.

\section{Real-time RT-PCR}

Total RNA was isolated using TRIzol Reagent (Invitrogen, Carlsbad CA, USA) and cDNA was synthetized using the Revert Aid First Strand cDNA Synthesis kit (Thermo Scientific, Rockford, IL, USA) according to manufacturers' instructions. Real-time PCR was performed with the StepOnePlus Real-Time PCR System (Applied Biosystems, USA), using SYBR Green (Fast SYBR Green Master Mix, Applied Biosystems, Warrington, UK), and specific primers for Ptafr (forward, 5'-AGC AGA GTT GGG CTA CCA GA-3' and reverse, 5'TGC GCA TGC TGT AAA ACT TC-3'), Lpcat2 (forward, 5'CCA GGT GGC ATT TAA GCT CT3' and reverse, 5’TCT TGG CAT ATT CTG 
Citation: Koga MM, Filgueiras LR, Jancar S, Rios FJ (2017) Platelet-Activation Factor Receptor Induces Interleukin 10 Production through

Page 4 of 9

GGT GC3'), Myds8 (forward, 5'- CTG TAA AGG CTT CTC GGA CTC-3' and reverse, 5'-GTG AGG ATA TAC TGA AGG AGC TG-3'), Trif (forward, 5'-GGT TCA CGA TCC TGC TCC TGA C-3' and reverse, 5'-GCT GGG CCT GAG AAC ACT CAA G-3'), Tirap (forward, 5'-AGT ATG GGG GAT GAA CGT G-3' and reverse, 5'AAC CCG ATT GAT GAG CC-3'), Cd36 (forward, 5'- TGC TGG AGC TGT TAT TGG TG-3' and reverse, 5'-TGG GTT TTG CAC ATC AAA GA-3'), III0 (forward, 5'CAG AGC CAC ATG CTC CTA GA3' and reverse, 5'TGT CCA GCT GGT CCT TTG TT3') and Gapdh (forward, 5'-AGG TCG GTG TGA ACG GAT TTG-3' and reverse, 5'TGT AGA CCA TGT AGT TGA GGT CA-3').

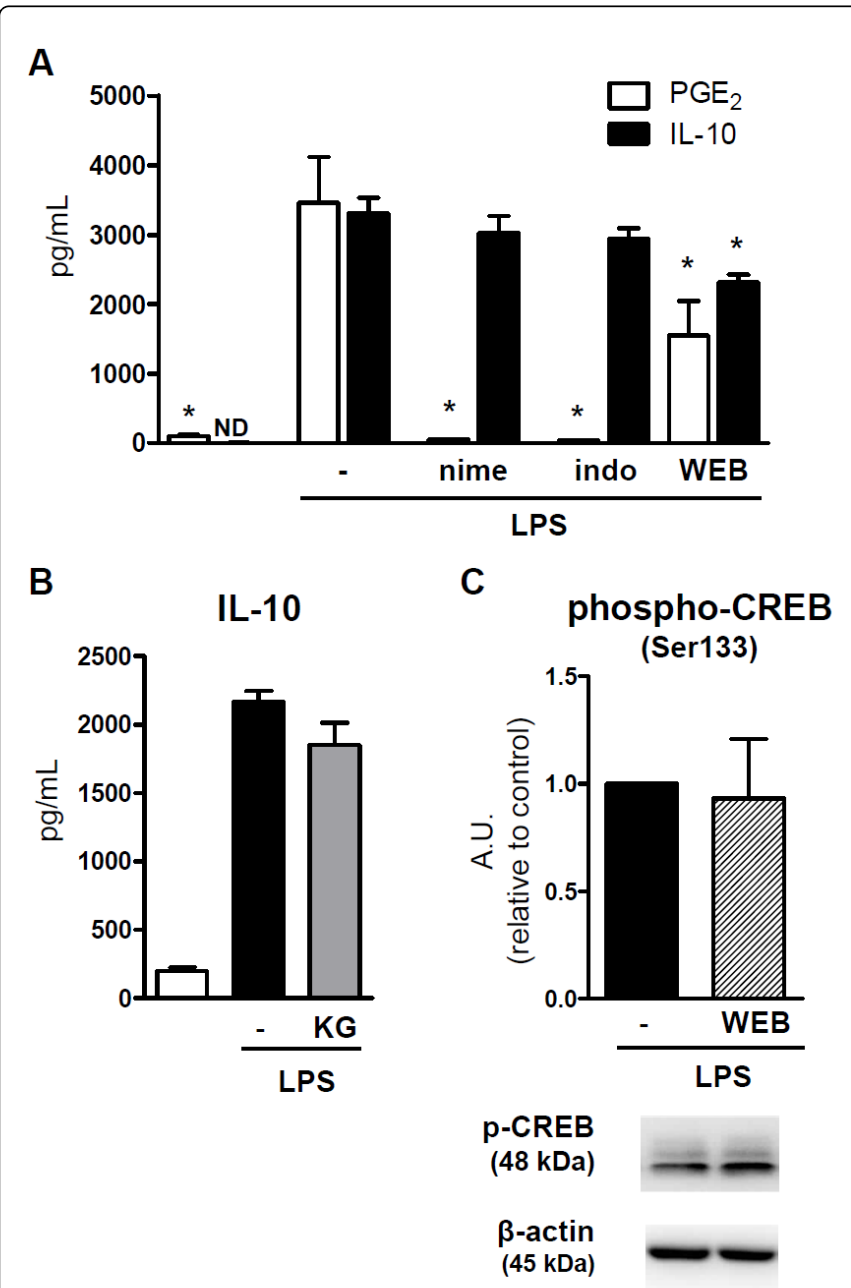

Figure 2: IL-10 production through PAFR in LPS-stimulated DCs is independent on CREB activation. DCs were pre-treated with nimesulide $(10 \mu \mathrm{M})$, indomethacin $(15 \mu \mathrm{M})$, WEB2086 $(50 \mu \mathrm{M})$, or KG-501 $(10 \mu \mathrm{M})$ for $30 \mathrm{~min}$. The production of PGE2 or IL-10 was assessed by ELISA in cultures supernatants after LPS stimulation for $24 \mathrm{~h}(\mathrm{~A}$ and B). DCs were treated with WEB2085 $(50 \mu \mathrm{M})$ for 30 min and stimulated with LPS $(1 \mu \mathrm{g} / \mathrm{mL})$. Lysates obtained after 30 min of stimulation were evaluated for phospho-CREB (Ser133) by Western blot (C). Densitometric analysis of phospho-CREB/ $\beta$-actin levels are expressed as mean \pm SEM of fold change of the vehicle control group. $(\mathrm{n}=3) .{ }^{\star} \mathrm{P}<0.05$ vs. untreated control group.
Relative gene expression was calculated using the $2^{-\Delta \Delta \mathrm{CT}}$ method as previously described [12]. Data were normalized by the housekeeping gene (Gapdh) and expressed as fold change relative to control.

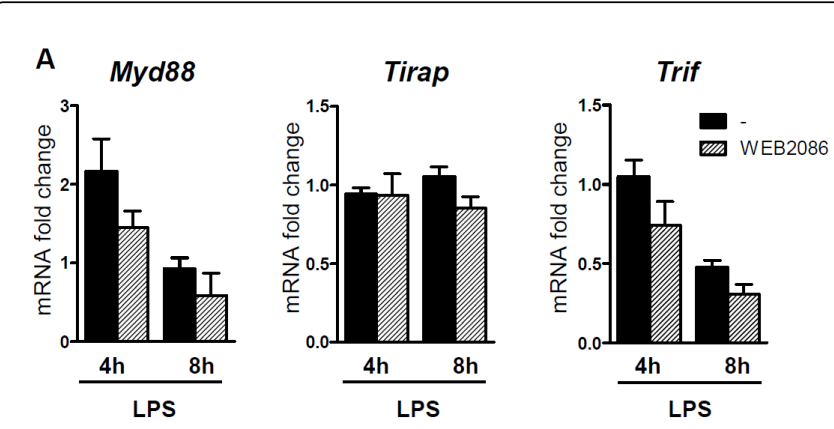

B
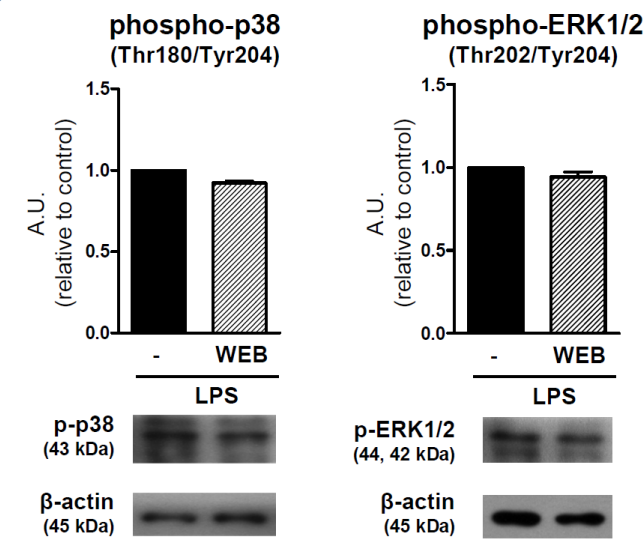

C

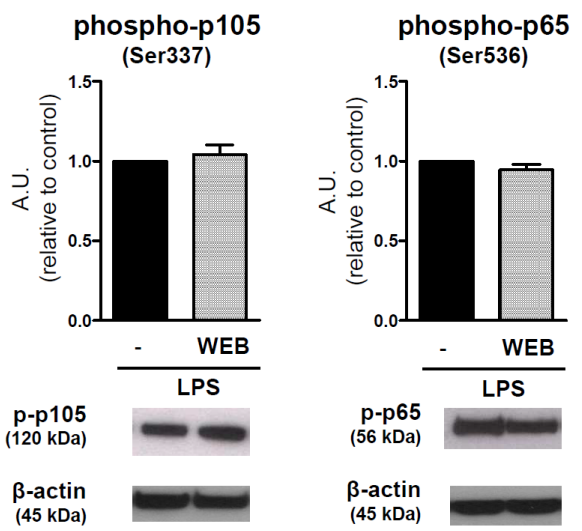

Figure 3: PAFR does not modulate TLR4 adaptor molecules (MyD88 and TRIF), phosphorylation of MAPKs, or NF-kB p105/50 or p65 subunits in LPS-stimulated DCs. DCs were pre-treated with WEB2086 $(50 \mu \mathrm{M})$ for $30 \mathrm{~min}$ and stimulated with LPS $(1 \mu \mathrm{g} / \mathrm{mL})$. Myd88, Trif, and Tirap gene expression was assessed by qPCR. Data are shown as fold change of the untreated control group (A). Lysates obtained after $30 \mathrm{~min}$ stimulation were evaluated by Western blot for phospho-p38 (Thr180/Tyr204) or phospho-ERK1/2 (Thr202/ Tyr204) (B), and phospho-p105 (Ser337) or phospho-p65 (Ser536) (C). Densitometric analyses of phospho-proteins/ $\beta$-actin levels are expressed as mean \pm SEM of fold change of the untreated control group. $(n=3)$. 


\section{Statistical analysis}

Results were presented as mean values \pm SEM. Statistical differences between mean values were determined by the one-way ANOVA, followed by the Newman-Keuls test or Student's $t$ test, using Graph Pad Prism 5.0 software (San Diego, CA, USA). P $<0.05$ values were considered as significant.

\section{Results}

\section{PAFR ligands are produced by LPS-stimulated DCs}

DCs were differentiated from murine bone-marrow by mrGM-CSF treatment for 6 days and stimulated with LPS $(1 \mu \mathrm{g} / \mathrm{mL})$. After $24 \mathrm{~h}$, cells were investigated for PAFR expression. Figures $1 \mathrm{~A}$ and $\mathrm{B}$ shows that PAFR expression (protein and mRNA, respectively) was detected at similar levels in both unstimulated and in LPS- stimulated DCs. In order to explore the generation of PAFR ligands, firstly we found that the LPS stimulus upregulated the gene expression for the inducible lyso-PAF acetyltransferase (Lpcat2), an enzyme involved for PAF biosynthesis (Figure 1C). The generation of PAFR ligands was investigated using the KB PAFR cell model system. PAFR-expressing KBP cells incubated with DCs lipid extracts showed IL-8 production compared to the vehicle that was maximized (3-fold) in LPSstimulated DCs (Figure 1D). IL-8 production by PAFR-deficient KBM cells stimulated with DCs lipid extracts was under detection limit (data not shown). The specificity of the experiment was verified in KBP and KBM cells stimulated with $100 \mathrm{nM}$ of methylcarbamyl-PAF (CPAF - a stable PAF analog) or $10 \mathrm{nM}$ of PMA. Figure 1E shows that KBP but not KBM cells produced IL-8 when stimulated with CPAF, while PMA induced similar levels of IL-8 production in both cell lines.

\section{LPS induces IL-10 production through PAFR and independent of $\mathrm{COX}-2 / \mathrm{PGE}_{2}$ axis}

LPS has been reported to induce $\mathrm{PGE}_{2}$ synthesis through COX-2 in DCs $[8,13,14]$. The secreted $\mathrm{PGE}_{2}$ could then act on its receptors EP2 and EP4 expressed on the cell membrane and increases the levels of cyclic adenosine monophosphate (cAMP) cellular [15]. In response, transcription factors such as CREB (the cAMP response element binding protein) bind to the Il10 promoter to induce IL-10 production. We have previously shown that PAFR activation contributes to the production of both $\mathrm{PGE}_{2}$ and IL-10 in LPS-stimulated DCs [8]. However, when DCs were pre-treated with the COX-2 non-selective inhibitor indomethacin $(15 \mu \mathrm{M})$ or the selective inhibitor nimesulide $(10 \mu \mathrm{M})$, no effects were observed in IL-10 levels; hence, the IL-10 production was not affected by endogenous $\mathrm{PGE}_{2}$, since $\mathrm{PGE}_{2}$ was completely suppressed by COX inhibitors. The blockage of PAFR with WEB2086 reduced both $\mathrm{PGE}_{2}$ and IL-10 LPS-induced production (Figure 2A), thus confirming our previous data [8].The requirement of CREB activation for IL-10 production was also excluded, since no changes in the IL-10 production were observed in LPS stimulated DCs in the presence of the CREB inhibitor KG-501 $(10 \mu \mathrm{M})$ (Figure $2 \mathrm{~B}$ ). Additionally, DCs pre-treatment with the PAFR antagonist WEB2086 (WEB-50 $\mu \mathrm{M}$ ) did not affect CREB phosphorylation (Figure 2C). These results indicate that IL-10 production by LPS stimulated DCs occurred through PAFR activation but independently of the COX-2/PGE 2 axis and CREB activation.
PAFR activation does not modulate TLR4 downstream adaptor molecules (MyD88 and TRIF) expression, MAPKs or NF- $\mathrm{KB}$ phosphorylation in LPS-stimulated DCs.

The intracellular signaling pathway activated by LPS for IL-10 production requires the activation of MyD88 and TRIF [1]. To check if the blockage of the PAFR was modulating the TLR4 adaptor proteins, DCs were treated with WEB2086 for 30 min and then stimulated with LPS $(1 \mu \mathrm{g} / \mathrm{mL})$ for 4 or $8 \mathrm{~h}$. Figure 3A shows that Myd88, Tirap, or Trif expressions were not affected in the absence of PAFR activation. Further downstream of TLR4 signaling, the mitogen activated protein kinases (MAPKs) and the nuclear factor- $\mathrm{\kappa B}(\mathrm{NF}-\mathrm{\kappa B})$ pathways are also activated in IL-10 induction. In fact, we have previously shown that PAFR agonists induced IL- 8 and IL-10 production through p38 and ERK1/2 activation in human macrophages [16]. To test if these effects also occurred in LPS-stimulated DCs, cells were pre-treated with WEB2086. Figure 3B shows that no differences in p38 or ERK-1/2 phosphorylation were detected in cells previously treated with WEB2086. In another study, our group has observed that the addition of CPAF to murine macrophages stimulated with LPS potentiated IL-10 production through the phosphorylation of NF- $\kappa B$ p105/50 [7]. To test the hypothesis that the blockage of the PAF signaling pathway would also affect the p105/50 subunit phosphorylation in DCs, cells were pre-treated with WEB2086 as above mentioned, and stimulated with LPS for $30 \mathrm{~min}$. However, this pathway was also excluded, since WEB2086 treatment did not alter p105/50 or p65 phosphorylation patterns (Figure 3C).
A

IL-10

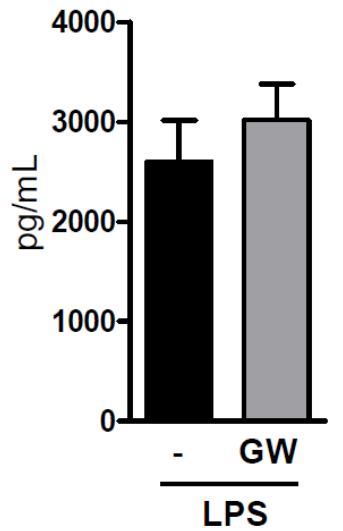

B

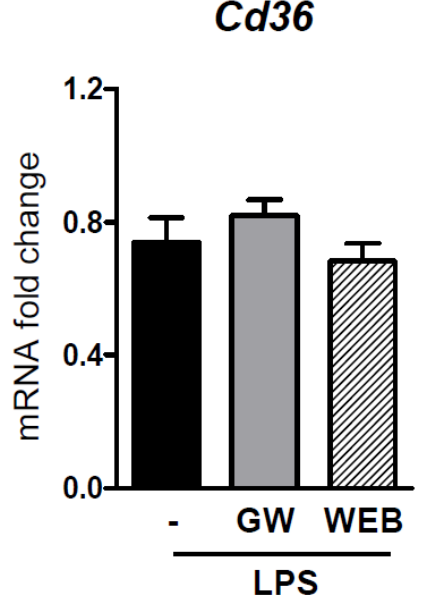

Figure 4: IL-10 production through PAFR in LPS-stimulated DCs is independent on PPAR $\gamma$ activation. DCs were pre-treated with WEB2086 $(50 \mu \mathrm{M})$ or GW9662 $(10 \mu \mathrm{M})$ for $30 \mathrm{~min}$ and stimulated with LPS $(1 \mu \mathrm{g} / \mathrm{mL})$. IL-10 production was assessed by ELISA in cultures supernatants after $24 \mathrm{~h}$ of stimulation (A). Cd36 gene expression was assessed by qPCR (B). Data are shown as fold change of the untreated control group. $(n=3)$.

\section{IL-10 production in LPS-stimulated DCs is independent of PPAR $\gamma$ activation}

The activation of the PAFR by oxidized Low Density Lipoprotein (oxLDL) was also shown to induce IL-10 and the peroxisome 
Citation: Koga MM, Filgueiras LR, Jancar S, Rios FJ (2017) Platelet-Activation Factor Receptor Induces Interleukin 10 Production through

proliferator-activated receptor $\gamma(\operatorname{PPAR} \gamma)$ expression upregulation in human and mouse macrophages [17]. In our experiments, however, the requirement of PPAR $y$ for IL-10 production was excluded, since the treatment with the PPAR $\gamma$ antagonist GW9662 $(10 \mu \mathrm{M})$ did not affect the IL-10 production by DCs (Figure 4A).

A

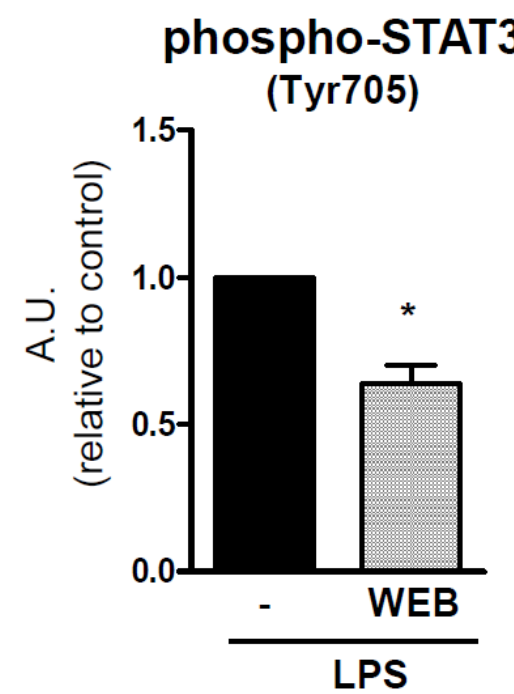

total-STAT3
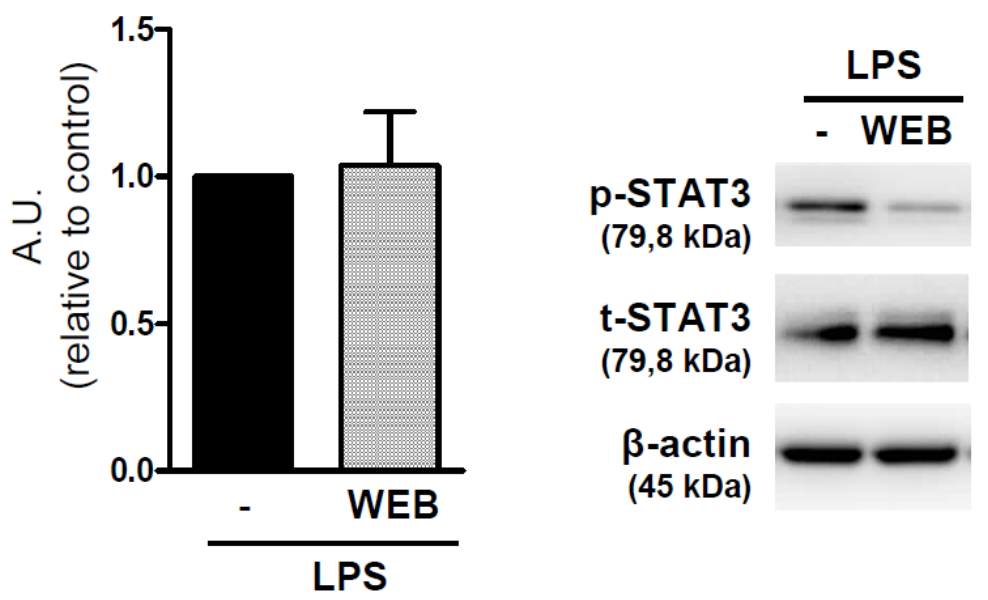

B

C

D

E

IL-10

(WT DCs)

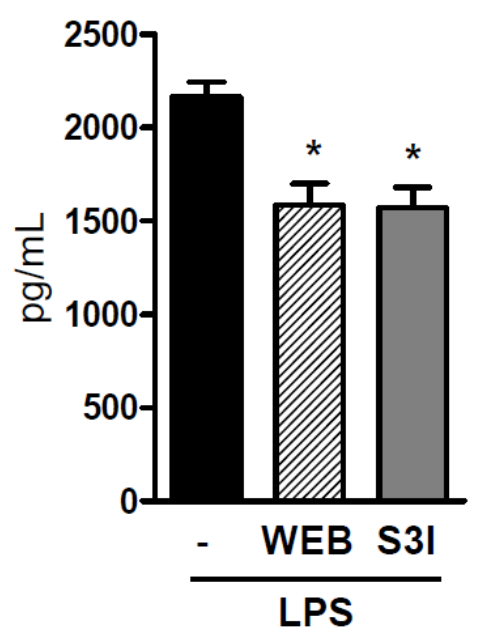

II10 (WT DCs)

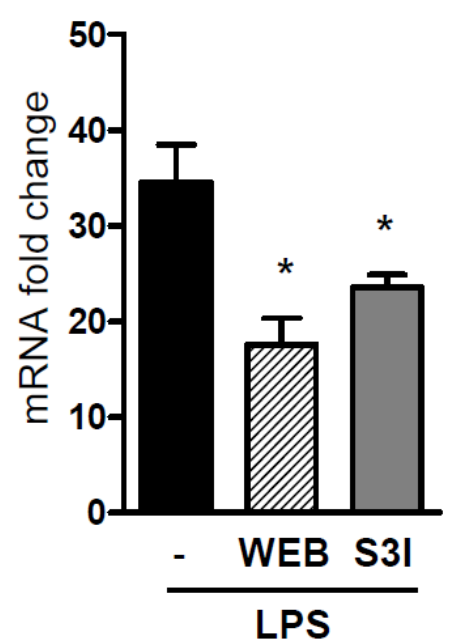

IL-10 (PAFR-KO DCs)

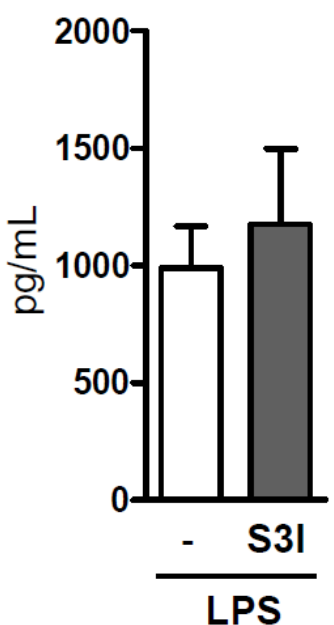

II10 (PAFR-KO DCs)

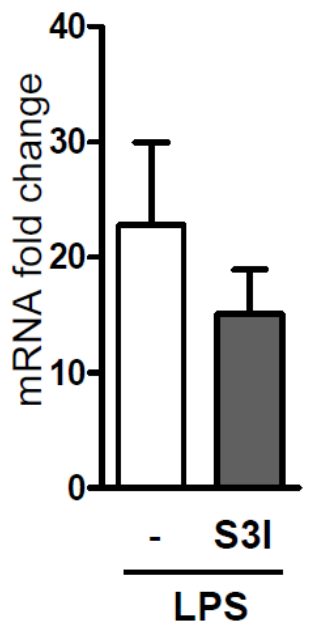

Figure 5: PAFR/STAT3 activation is required for IL-10 production in LPS-stimulated DCs. Bone marrow-differentiated DCs of wild-type (WT) or PAFR deficient (PAFR-KO) mice were pre-treated with WEB2086 (50 $\mu \mathrm{M})$ or S3I-201 $(50 \mu \mathrm{M})$ for 30 min and stimulated with LPS (1 $\mu \mathrm{g} / \mathrm{mL}$ ). Lysates obtained from WT DCs were evaluated for phospho-STAT3 (Tyr705) or total-STAT3 by Western blot. Densitometric analyses of phospho-STAT3 $/ \beta$-actin or total-STAT3/ $\beta$-actin levels are expressed as fold change of the untreated control group (A). IL-10 production was assessed by ELISA in cultures supernatants and 1110 gene expression was assessed by qPCR from WT (B and C) and PAFR-KO (D and E) after $24 \mathrm{~h}$ of stimulation. Data are shown as fold change of the untreated control group. $(\mathrm{n}=3)$. ${ }^{*} \mathrm{P}<0.05$ vs. untreated control group. 
The non-requirement of PPAR $\gamma$ activation in LPS-stimulated DCs was also confirmed by the expression of PPAR $\gamma$-inducible gene for the scavenger receptor $C D 36$ (encoded by Cd36), which was not affected by PPAR $\gamma$ or PAFR antagonists (Figure 4B). Altogether, these data show that DCs signaling pathway for IL-10 production induced by LPS requires different intracellular molecules than the ones observed in macrophages.

\section{PAFR/STAT3 activation is required for IL-10 production in LPS-stimulated DCs}

The activation of signal transducer and activator of transcription 3 (STAT3) is classically described as a regulator of cells response to IL-10 and IL-6. Moreover, STAT3 can be activated through TLR4 signalling [18] and also through PAFR $[19,20]$. We then hypothesized that the signal induced by PAFR ligands produced during LPS stimulation could synergize with the already LPS-induced STAT3 activation, amplifying IL-10 induction. Thus, we first checked the STAT3 phosphorylation in LPS-stimulated DCs. Figure 5A shows that the STAT3 phosphorylation induced by LPS was clearly reduced in the presence of PAFR antagonist. The necessity of STAT3 activation was confirmed in DCs treated with the STAT3 inhibitor S3I-201 $(50 \mu \mathrm{M})$, which reduced the LPS-induced IL-10 production to the same levels of WEB-treated DCs (Figure 5B). Same pattern was observed in $I 110$ gene expression (Figure 5C). To confirm the PAFR-STAT3-IL-10 axis, DCs differentiated from PAFR-KO mice were pre-treated with S3I-201 (50 $\mu \mathrm{M})$ followed by LPS stimulation. Figure $5 \mathrm{D}$ and $\mathrm{E}$ show that in the absence of the PAF receptor signalling, S3I-201 treatment did not modulate IL-10 protein or gene expression. Altogether, these results indicate that part of the IL-10 production in DCs induced by LPS stimulation is dependent on PAFR/STAT3 activation.

\section{Discussion}

Major findings from the present study demonstrate that PAFR activation enhance the IL-10 production induced by LPS stimulation in dendritic cells. Molecular mechanisms underlying these effects involve the production of PAFR ligands and STAT3 activation. Moreover, these mechanisms are different from what we previously observed in macrophages, since they are independent on $\mathrm{PGE}_{2}$ production, PPAR $\gamma$, or NF-kB activation $[7,16,17]$.

IL-10 is an anti-inflammatory cytokine produced by macrophages, DCs, and lymphocytes, and is able to regulate the activation of innate and adaptive immune responses. This cytokine is also a marker of alternatively activated macrophages which are involved in repair mechanisms, by inducing fibroblasts activation and collagen production [21]. In previous studies, we described the effect of the PAFR blockage in DCs during maturation induced by LPS. A pretreatment with a PAFR antagonist reduced the IL-10 and $\mathrm{PGE}_{2}$ secretion by DCs and increased their ability in promoting $\mathrm{T}$ cell proliferation and Th1-like adaptive response in vitro and in vivo $[6,8]$. Those experimental observations suggested that LPS stimulation induced the production of PAFR ligands that would act in a paracrine/ autocrine way. Molecular mechanisms involved in PAFR ligands generation through TLR4 activation were already observed in macrophages [22], however in DCs they were still elusive. To study the PAFR activation in LPS stimulated DCs, we first confirmed that PAFR is expressed by DCs and is not modulated by LPS stimulation in the first $24 \mathrm{~h}$. In previous data, we have shown that PAFR expression was only reduced after $48 \mathrm{~h}$ of LPS [8]. These results are in line to another report that showed PAFR expression reduction after $48 \mathrm{~h}$ and undetectable expression after $96 \mathrm{~h}$ in human monocyte-derived DCs stimulated with LPS [23]. In addition, we found that in DCs, LPS induced the expression of the inducible lyso-PAF acetyltransferase (LPCAT2), which is the key enzyme involved in PAF biosynthesis. LPCAT2 had already been described to be activated by LPS in mouse peritoneal macrophages and was also shown to be regulated by PAFR activation [22,24]. Indeed, the generation of PAFR ligands was confirmed to be produced in DCs and maximized by LPS stimuli.

We then sought to understand the mechanisms involved in the IL-10 production related to TLR4/PAFR activation in DCs. The increase in IL-10 production through PAFR activation in LPSstimulated DCs could be a result of the activation induced by COX-2/PGE 2 , which is also formed upon stimulation with TLR4 ligands and has been shown to be responsible for IL-10 production in DCs and in macrophages stimulated with zymozan and apoptotic cells $[25,26]$. Molecular mechanisms described for the production of IL-10 were also dependent on CREB activation by signalling pathways that involved EP receptors and the formation of cAMP and PKA activation [25]. However, our data suggested a different pathway, since the cell treatment with the inhibitors of COX-2 or CREB did not affect the production of IL-10, although the $\mathrm{PGE}_{2}$ production was completely abrogated by COX-2 inhibitors.

The regulation of IL-10 production by macrophages and DCs in response to TLR4 ligands can also be dependent on MAPK activation. In fact, we have previously observed that in macrophages the PAFR activation by oxidized Low Density Lipoprotein (oxLDL) increased LPS-induced IL-10 production and phosphorylation of ERK1/2 and p38 [16,17]. In the present study, however, the blockage of PAFR by antagonist treatment reduced IL-10 production but did not affect MAPK kinases phosphorylation in DCs. Following the TLR4 stimulation, we also checked for adaptor proteins expression and phosphorylation of NF- $\kappa B$ subunits p65 and p105/50, but when the PAFR was blocked by the antagonist WEB2086 none of these TLR4induced signalling pathways were affected.

Another possible pathway to be tested was the activation of the PPAR $\gamma$, a nuclear receptor capable of modulating inflammatory responses. PPAR $\gamma$ was described to induce anti-inflammatory M2 phenotype macrophages, and also related to PAFR activation and IL-10 release [17,27]. In DCs, the pre-treatment with the PPAR $\gamma$ antagonist GW9662 did not affect the LPS-induced IL-10 production. In addition, $\mathrm{CD} 36$, which is a scavenger receptor upregulated by PPAR $\gamma$ activation in macrophages was not, affected by the treatment with either PAFR or PPAR $\gamma$ antagonists. These results are different from the ones observed in DCs stimulated with zymosan, where there was a clear reduction of IL-10 production upon PPAR $\gamma$ inhibition [28].

DCs and macrophages produce significant amounts of IL-10 upon stimulation with TLR4 ligands and in macrophages we and others demonstrated that the IL-10 production is a result of a complex signalling pathway that involves mechanisms dependent on NF-kB, MAPK [7], PPAR $\gamma$ [29], CREB [30], and COX-2 [13]. Interestingly, none of these intracellular signaling pathways were involved in the IL-10 production enhanced by PAFR activation in DCs, which according to our results, STAT3 is a major player. These results are also different to what we previously observed in macrophages, where the IL-10 production enhanced by PAFR activation was not dependent on STAT3 [7]. Although macrophages and DCs are myeloid derived cells, they express different membrane markers that reflect in their phenotype and functionality. Macrophages have high expression of F4/80, CD36, and TLRs, whereas DCs present high expression of 
MHCII, costimulatory molecules (CD80, CD86, CD40), TLRs, and have properties of antigen presenting cells higher than macrophages. We showed before that CD36 is a scavenger receptor able to assemble with PAFR in lipid rafts to interact with PAFR ligands and enhance the production of IL-10 by macrophages [17,31]. These effects were abrogated in the presence of PAFR antagonists or anti-CD36 blocking antibody [17]. Comparing to macrophages, DCs express less CD36 [32, 33] and TLR4 in the membrane [34], thus these mechanisms above mentioned for macrophages are less significant in DCs. Additionally, it was demonstrated that PAFR activation induced CD36 expression in macrophages by mechanisms dependent on PPAR $\gamma$ [17]. In our present study, these mechanisms were also excluded, since no differences in CD36 expression were found in DC pre-treated with PAFR antagonist. Data present in the literature describe important role of PPAR $\gamma$ and CREB activity in DCs stimulated with LPS. The discrepancy of our results could be justified by the different experimental protocols used, most likely the one employed to differentiate BM-DCs: we only used GM-CSF in the culture medium, whereas others used GM-CSF plus IL-4. These protocols were shown to generate DC populations with different phenotypes which would activate distinct pathways in response to LPS [35]. In fact, DC differentiated in the presence of IL- 4 presented augmented PPAR $\gamma$ activity through mechanisms dependent on STAT6 activation [36], which was also observed to decrease the IL-10 production and increase IL-12 [37], therefore generating a DC population different than we used in our present study.

The activation of STAT3 is classically described as a regulator of cells response to IL-10 binding to its receptor, and inducing its own gene expression [38]. This was already observed in human monocytederived DCs and macrophages $[39,40]$. In addition, STAT3 can also be activated via TLR4 [18], indicating a IL-10 induction pathway independent of IL-10 receptor/STAT3 activation. Indeed, STAT3 phosphorylation was also observed as consequence of PAFR activation in monocytic cell lines [19], in HUVECs [20], and in intestinal epithelial cells [41]. Our results indicated that the blockage of PAFR during LPS stimulation, significantly reduced STAT3 Tyr705 phosphorylation. When DCs were treated with the STAT3 inhibitor S3I-201, we observed decreased production of IL-10 and gene expression in similar levels as observed when the PAFR antagonist WEB2086 was added to DCs. To confirm the requirement of PAFR activation to the TLR4/STAT3 pathway to IL-10 production, DCs derived from PAFR-KO mice were treated with STAT3 inhibitor and stimulated with LPS. Both IL-10 protein and gene expression levels were not altered by the treatment and were found in similar levels as observed in wild type DCs treated with PAFR antagonist. A recently report has shown that IL-10 production in human monocyte-derived DCs was increased by zymosan stimulus, which induced STAT3 Tyr705 phosphorylation. It was also observed that when cells were stimulated in the presence of WEB2086, STAT3 Tyr705 phosphorylation was reduced [42]. These data reinforce our findings in associating PAFR/STAT3 activation in IL-10 production in DCs. Moreover, it strengthens the importance of the PAFR activation in DCs phenotype and function.

Based on these data, we propose that PAFR ligands are produced by DCs stimulated with LPS and that it engages the PAFR in DC membranes to potentiate LPS-induced IL-10 by mechanisms dependent on STAT3 activation. The production of IL-10, together with $\mathrm{PGE}_{2}$ as we previously demonstrated, contributes to the establishment of a regulatory phenotype in DCs. These data add some explanation to our previous studies where we showed that the blockage of PAFR enhance the adaptive immune response. In addition, this study brings additional molecular mechanisms by which PAFR contributes to IL-10 production by dendritic cells.

\section{Conflict of Interests}

The authors declare no conflict or competing financial interests.

\section{Acknowledgement}

The authors are grateful to Marlise Montes and Silvana Silva for technical support. This work was supported by Fundação de Amparo à Pesquisa do Estado de São Paulo (FAPESP) and Conselho Nacional de Desenvolvimento Científico e Tecnológico (CNPq).

Contract grant sponsor: FAPESP; Contract grant number: 2013/15719-0.

\section{References}

1. Saraiva M, O'Garra A (2010) The regulation of IL-10 production by immune cells. Nat Rev Immunol 10: 170-181.

2. Medzhitov R (2007) Recognition of microorganisms and activation of the immune response. Nature 449: 819-826.

3. Harizi H, Gualde N (2005) The impact of eicosanoids on the crosstalk between innate and adaptive immunity: the key roles of dendritic cells. Tissue Antigens 65: 507-514.

4. Shindou H, Ishii S, Uozumi N, Shimizu T (2000) Roles of cytosolic phospholipase $\mathrm{A}(2)$ and platelet-activating factor receptor in the Cainduced biosynthesis of PAF. Biochem Biophys Res Commun 271: 812-817.

5. Marrache AM, Gobeil F, Bernier SG, Stankova J, Rola-Pleszcynski M, et al. (2002) Proinflammatory gene induction by platelet-activating factor mediated via its cognate nuclear receptor. J Immunol 169: 6474-6481.

6. Koga MM, Bizzarro B, Sá-Nunes A, Rios FJ, Jancar S (2016) Boosting Adaptive Immunity: A New Role for PAFR Antagonists. Sci Rep 6: 39146.

7. Ishizuka EK, Filgueiras LR, Rios FJ, Serezani CH, Jancar S (2016) PAFR activation of NF-kB p65 or p105 precursor dictates pro- and antiinflammatory responses during TLR activation in murine macrophages. Sci Rep 6: 32092.

8. Koga MM, Bizzarro B, Sá-Nunes A, Rios FJ, Jancar S (2013) Activation of PAF-receptor induces regulatory dendritic cells through PGE2 and IL-10. Prostaglandins Leukot Essent Fatty Acids 89: 319-326.

9. Kalinski P (2012) Regulation of immune responses by prostaglandin E2. J Immunol 188: 21-28.

10. Ishii S, Kuwaki T, Nagase T, Maki K, Tashiro F, et al. (1998) Impaired anaphylactic responses with intact sensitivity to endotoxin in mice lacking a platelet-activating factor receptor. J Exp Med 187: 1779-1788.

11. Pei Y, Barber LA, Murphy RC, Johnson CA, Kelley SW, et al. (1998) Activation of the epidermal platelet-activating factor receptor results in cytokine and cyclooxygenase-2 biosynthesis. J Immunol 161: 1954-1961.

12. Livak KJ, Schmittgen TD (2001) Analysis of relative gene expression data using real-time quantitative PCR and the 2(-Delta Delta C(T)) Method. Methods 25: 402-408.

13. Harizi H, Juzan M, Pitard V, Moreau JF, Gualde N (2002) Cyclooxygenase-2-issued prostaglandin e(2) enhances the production of endogenous IL-10, which down-regulates dendritic cell functions. J Immunol 168: 2255-2263.

14. Fogel-Petrovic M, Long JA, Knight DA, Thompson PF, Upham JW (2004) Activated human dendritic cells express inducible cyclo-oxygenase and synthesize prostaglandin E2 but not prostaglandin D2. Immunol Cell Biol 82: $47-54$.

15. Harizi H, Grosset C, Gualde N (2003) Prostaglandin E2 modulates dendritic cell function via EP2 and EP4 receptor subtypes. J Leukoc Biol 73: 756-763. 
Citation: Koga MM, Filgueiras LR, Jancar S, Rios FJ (2017) Platelet-Activation Factor Receptor Induces Interleukin 10 Production through STAT3 Activation in Dendritic Cells. J Immuno Biol 2: 123. doi:10.4172/2476-1966.1000123

Page 9 of 9

16. Rios FJ, Koga MM, Ferracini M, Jancar S (2012) Co-stimulation of PAFR and CD36 is required for oxLDL-induced human macrophages activation. PLoS One 7: e36632.

17. Rios FJ, Koga MM, Pecenin M, Ferracini M, Gidlund M, et al. (2013) Oxidized LDL Induces Alternative Macrophage Phenotype through Activation of CD36 and PAFR. Mediators Inflamm 2013: 198193.

18. Carl VS, Gautam JK, Comeau LD, Smith MF (2004) Role of endogenous IL-10 in LPS-induced STAT3 activation and IL-1 receptor antagonist gene expression. J Leukoc Biol 76: 735-742.

19. Lukashova V, Asselin C, Krolewski JJ, Rola-Pleszczynski M, Stanková J (2001) G-protein-independent activation of Tyk2 by the plateletactivating factor receptor. J Biol Chem 276: 24113-24121.

20. Deo DD, Axelrad TW, Robert EG, Marcheselli V, Bazan NG, et al. (2002) Phosphorylation of STAT-3 in response to basic fibroblast growth factor occurs through a mechanism involving platelet-activating factor, JAK-2, and $\mathrm{Src}$ in human umbilical vein endothelial cells. Evidence for a dual kinase mechanism. J Biol Chem 277: 21237-21245.

21. Gordon S (2003) Alternative activation of macrophages. Nat Rev Immunol 3: 23-35.

22. Shindou H, Ishii S, Yamamoto M, Takeda K, Akira S, et al. (2005) Priming effect of lipopolysaccharide on acetyl-coenzyme A:lyso-plateletactivating factor acetyltransferase is MyD88 and TRIF independent. J Immunol 175: 1177-1183.

23. Dichmann S, Rheinen H, Panther E, Herouy Y, Czech W, et al. (2000) Downregulation of platelet-activating factor responsiveness during maturation of human dendritic cells. J Cell Physiol 185: 394-400.

24. Doebber TW, Wu MS (1987) Platelet-activating factor (PAF) stimulates the PAF-synthesizing enzyme acetyl-CoA:1-alkyl-sn-glycero-3phosphocholine O2- acetyltransferase and PAF synthesis in neutrophils. Proc Natl Acad Sci USA. 84: 7557-7561.

25. Alvarez Y, Municio C, Alonso S, Sánchez-Crespo M, Férnandez N (2009) The induction of IL-10 by zymosan in dendritic cells depends on CREB activation by the coactivators CREB-binding protein and TORC2 and autocrine PGE2. J Immunol 183: 1471-1479.

26. Fadok VA, Bratton DL, Konowal A, Freed PW, Westcott JY, et al. (1998) Macrophages that have ingested apoptotic cells in vitro inhibit proinflammatory cytokine production through autocrine/paracrine mechanisms involving TGF-beta, PGE2, and PAF. J Clin Invest 101: 890-898.

27. Bouhlel MA, Derudas B, Rigamonti E, Dièvart R, Brozek J, et al. (2007) PPARgamma activation primes human monocytes into alternative M2 macrophages with anti-inflammatory properties. Cell Metab 6: 137-143.

28. Municio C, Hugo E, Alvarez Y, Alonso S, Blanco L, et al. (2011) Apoptotic cells enhance IL-10 and reduce IL-23 production in human dendritic cells treated with zymosan. Mol Immunol 49: 97-106.
29. Ferreira AE, Sisti F, Sônego F, Wang S, Filgueiras LR, et al. (2014) PPAR$\gamma / \mathrm{IL}-10$ axis inhibits MyD88 expression and ameliorates murine polymicrobial sepsis. J Immunol 192: 2357-2365.

30. Wen AY, Sakamoto KM, Miller LS (2010) The role of the transcription factor CREB in immune function. J Immunol 185: 6413-6419.

31. Rios FJ, Ferracini M, Pecenin M, Koga MM, Wang Y, et al. (2013) Uptake of oxLDL and IL-10 production by macrophages requires PAFR and CD36 recruitment into the same lipid rafts. PLoS One 8: e76893.

32. Misharin AV, Morales-Nebreda L, Mutlu GM, Budinger GR, Perlman H (2013) et al., Flow cytometric analysis of macrophages and dendritic cell subsets in the mouse lung. Am J Respir Cell Mol Biol 49: 503-510.

33. Drage MG, Pecora ND, Hise AG, Febbraio M, Silverstein RL, et al. (2009) TLR2 and its co-receptors determine responses of macrophages and dendritic cells to lipoproteins of Mycobacterium tuberculosis. Cell Immunol 258: 29-37.

34. Vaure C, Liu Y (2014) A comparative review of toll-like receptor 4 expression and functionality in different animal species. Front Immunol 5: 316

35. Labeur MS, Roters B, Pers B, Mehling A, Luger TA, et al.( 1999) Generation of tumor immunity by bone marrow-derived dendritic cells correlates with dendritic cell maturation stage. J Immunol 162: 168-175.

36. Szanto A, Balint BL, Nagy ZS, Barta E, Dezso B, et al. (2010) STAT6 transcription factor is a facilitator of the nuclear receptor PPAR $\gamma$ regulated gene expression in macrophages and dendritic cells. Immunity 33: 699-712.

37. Yao Y, Li W, Kaplan MH, Chang CH (2005) Interleukin (IL)-4 inhibits IL-10 to promote IL-12 production by dendritic cells. J Exp Med 201: 1899-1903.

38. Benkhart EM, Siedlar M, Wedel A, Werner T, Ziegler-Heitbrock HW (2000) Role of Stat3 in lipopolysaccharide-induced IL-10 gene expression. J Immunol 165: 1612-1617.

39. Corinti S, Albanesi C, Ia Sala A, Pastore S, Girolomoni G (2001) Regulatory activity of autocrine IL-10 on dendritic cell functions. J Immunol 166: 4312-4318.

40. Staples KJ, Smallie T, Williams LM, Foey A, Burke B, et al. (2007) IL-10 induces IL-10 in primary human monocyte-derived macrophages via the transcription factor Stat3. J Immunol 178: 4779-4785.

41. Soliman A, Michelsen KS, Karahashi H, Lu J, Meng FJ, et al. (2010) Platelet-activating factor induces TLR4 expression in intestinal epithelial cells: implication for the pathogenesis of necrotizing enterocolitis. PLoS One 5: e15044.

42. Rodríguez M, Márquez S, de la Rosa JV, Alonso S, Castrillo A, et al. (2017) Fungal pattern receptors down-regulate the inflammatory response by a cross-inhibitory mechanism independent of interleukin-10 production. Immunology 150: 184-198. 